PORTZ A; MARTINS CAC; LIMA E; ZONTA E. 2006. Teores e acúmulo de nutrientes durante o ciclo da mandioquinha-salsa em função da aplicação de nitrogênio, fósforo e potássio. Horticultura Brasileira 24: 329-333.

\title{
Teores e acúmulo de nutrientes durante o ciclo da mandioquinha-salsa em função da aplicação de nitrogênio, fósforo e potássio
}

\author{
Adriano Portz ${ }^{1}$; Carla Andréia da Cunha Martins²; Eduardo Lima ${ }^{2}$; Everaldo Zonta ${ }^{2}$ \\ ${ }^{1}$ UFF-Dep ${ }^{\text {to }}$ TAA, Av. dos Trabalhadores, 420 Vila Sta. Cecília, 27255-125 Volta Redonda-RJ; ${ }^{2}$ UFRRJ-IA-Dep ${ }^{\text {to }}$ Solos, BR 465, km 7 , \\ 23890-000 Seropédica-RJ; E-mail: aportz@metal.eeimvr.uff.br; edulima@ufrrj.br
}

\section{RESUMO}

Apesar da cultura da mandioquinha-salsa (Arracacia xanthorrhiza Bancroft) ser cultivada há bastante tempo no País, informações referentes aos seus aspectos nutricionais e seu desenvolvimento no campo são ainda incipientes. $\mathrm{O}$ estudo dos teores de nutrientes e seu comportamento ao longo do ciclo ontogenético é importante para se estabelecer uma correta recomendação de fertilizantes, níveis críticos e conseqüentemente uma maior produção de raízes comerciais. Com o objetivo de avaliar os teores e o acúmulo de macro e micronutrientes na cultura durante seu desenvolvimento, foi realizado um experimento de campo com três níveis de $\mathrm{N}, \mathrm{P}$ e $\mathrm{K}$, na região de Nova Friburgo-RJ, utilizando um delineamento experimental de blocos ao acaso com quatro repetições. Foram realizadas quatro amostragens ao longo do ciclo da cultura e coletadas três partes da planta (folhas, propágulo e raízes), observando-se ao longo do tempo o comportamento dos teores e o acúmulo dos nutrientes nessas partes. Não foi observada correlação dos teores de nutrientes nas partes da planta, nas épocas de amostragem, com a produção de raízes comerciais. Maior acúmulo de nutrientes foi observado entre os 150 e 210 DAT nas folhas, aos 210 DAT nos propágulos e aos 300 DAT nas raízes. Os teores foram influenciados pelos tratamentos aplicados no plantio do experimento, mas não foi observada resposta no aumento de produção de raízes comerciais.

Palavras-chave: Arracacia xanthorrhiza Bancroft, nutrição mineral, adubação NPK.

\section{ABSTRACT}

Levels and accumulation of nutrients in the cycle of peruvian carrot with application of nitrogen, phosphorus and potassium

The arracacha (Arracacia xanthorrhiza Bancroft) culture, in spite of already being cultivated for a long time in Brazil, has still low information on its nutritional aspect, and the field development. The study of the nutrient distribution inside the plant and their behavior along the vegetative cycle is important to establish a correct fertilizer recommendation, and to provide nutritional information, besides to obtain best commercial root production. The macro and micro nutrient levels and accumulation in the crop were evaluated through a field experiment accomplished with three levels of $\mathrm{N}, \mathrm{P}$, and $\mathrm{K}$, in Nova Friburgo, Rio de Janeiro State, using a random blocks experimental design with four repetitions. Four sample periods were observed along the crop cycle, where three sections of the plant (leaves, corm, and roots) were collected; also, changes in nutrient levels of the plant sections were monitored along the time periods. Correlation of the nutrient levels was not observed in the plant parts, in the sampling times, with the commercial roots production. Larger accumulations of the nutrients were observed between the 150 and 210 DAT in the leaves, 210 DAT in the corm and 300 DAT in the roots. The levels were influenced by the applied treatments, but an answer was not observed in the increase of commercial roots production.

Keywords: Arracacia xanthorrhiza, mineral nutrition, NPK fertilization.

(Recebido para publicação em 12 de julho de 2005; aceito em 31 de agosto de 2006)

\begin{abstract}
A área cultivada com mandioquinhasalsa vem aumentando em várias regiões do País, contribuindo para uma maior oferta desta raiz, que ainda é pouco conhecida, apesar de ser rica em nutrientes e de fácil digestibilidade. Segundo Pereira (1995), a mandioquinha-salsa é um alimento essencialmente energético, excelente fonte de cálcio, fósforo e ferro e, entre as vitaminas presentes, ressalta-se a vitamina $\mathrm{A}$, que pode atingir 6800 U.I.
\end{abstract}

Além das raízes, a parte aérea da mandioquinha também pode ser utilizada na alimentação humana e animal, como ocorre nos países Andinos, as folhas mais novas são usadas na forma de saladas e sopas. O propágulo (coroa + rebentos) possui maiores quantidades de nutrientes se comparados às raízes de reserva e, segundo Câmara (1984), também tem um melhor balanço de aminoácidos, indicando que esta parte da planta também pode ser utilizada na alimentação, apesar do maior teor de celulose quando comparada às raízes.

A pesquisa sobre a nutrição da cultura ainda é incipiente e há dificuldades para recomendação de adubação para a planta nos diferentes locais de cultivo e condições de manejo. A aplicação de nitrogênio e fósforo não apresenta uniformidade de resposta com a produção de raízes comerciais, mas respostas distintas em vários ambientes. Segundo Mesquita et al. (1996), não houve resposta na produção de raízes comerciais à adubação nitrogenada, mas houve res- posta quadrática para a aplicação de fósforo. Silva et al. (1966) observaram que doses maiores de $50 \mathrm{~kg} \cdot \mathrm{ha}^{-1}$ de nitrogênio contribuíram para o decréscimo, enquanto o fósforo proporcionou um aumento linear na produção de raízes comerciais. Del Valle Junior et al. (1995) recomendam a aplicação de até 135 kg.ha-1 de N para se obter uma boa produção sem, contudo, terem observado resposta com a aplicação de diferentes níveis de $\mathrm{P}, \mathrm{K}$ e Mg. Segundo Zárate et al. (2001), o aumento na dose de fósforo aplicado ao solo proporcionou um melhor balanço nutricional nas plantas, contribuindo para uma menor perda de peso das raízes após a colheita. A deficiência de cálcio e magnésio na cultura altera sobremaneira o desenvolvimento 


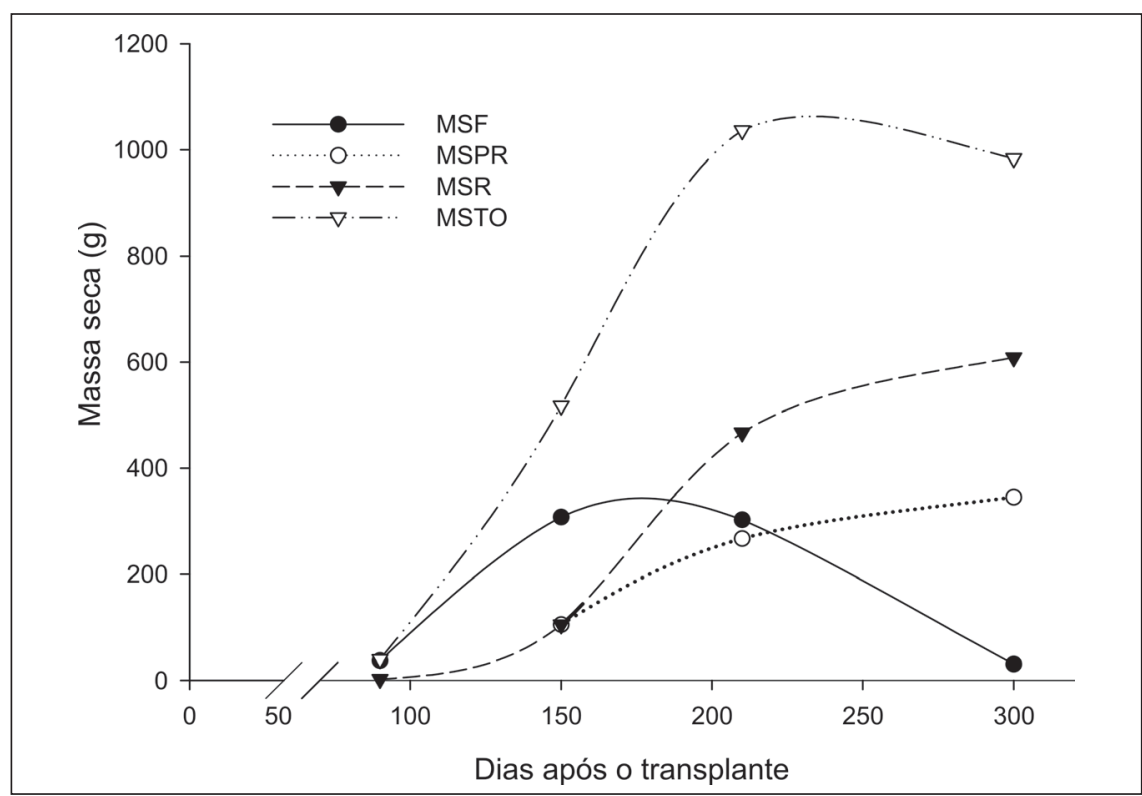

Figura 1. Comportamento do acúmulo de massa seca total da planta (MSTO), massa seca de folhas (MSF), massa seca do propágulo (MSPR) e massa seca das raízes (MSR) durante o ciclo da mandioquinha-salsa. Seropédica, UFRRJ, 2001.

da planta e os teores dos demais nutrientes (Câmara, 1990). A adubação orgânica é importante na cultura, pois melhora não só aspectos químicos, mas também a estrutura do solo, o que é fundamental para culturas que possuem a parte comercial subterrânea, como é o caso da mandioquinha-salsa.

O estudo dos teores de nutrientes na planta, assim como em suas partes, é de grande importância para uma melhor recomendação do manejo da adubação na cultura, tanto para áreas produtoras como em novas áreas de cultivo, evitando o desperdício de fertilizantes ou deficiências nutricionais ao longo do seu ciclo, contribuindo para um melhor manejo e ciclagem de nutrientes.

Com o objetivo de estudar o comportamento dos teores de nutrientes e o acúmulo de nutrientes na planta durante o ciclo da cultura, procurando obter informações sobre a exigência nutricional em seus estágios fenológicos, desenvolveu-se o presente trabalho.

\section{MATERIAL E MÉTODOS}

O experimento foi instalado em 1999 na Fazenda Santo Antônio de Estrela, Rio Grande de Cima, terceiro distrito de Nova Friburgo-RJ, com altitude média de $1000 \mathrm{~m}$, precipitação pluviométrica média de $1200 \mathrm{~mm}$ anuais e temperatura média anual de $18,9^{\circ} \mathrm{C}$. O solo classificado como NITOSSOLO VERMELHO distrófico (EMBRAPA, 1999), com $12 \%$ de declividade, e a camada de 0-20 $\mathrm{cm}$ de profundidade, antes do início da instalação do experimento, apresentava as seguintes características químicas: $\mathrm{pH}$ em água $(1: 2,5)=6,0 ; \mathrm{Al}^{+3}=$ $0,3 \mathrm{cmol} \cdot \mathrm{kg}^{-1} ; \mathrm{H}+\mathrm{Al}=18,3 \mathrm{cmol} \cdot \mathrm{kg}^{-1}$; $\mathrm{Mg}^{+2}=0,9 \mathrm{cmol} \cdot \mathrm{kg}^{-1} ; \mathrm{Ca}^{+2}=1,8$ $\mathrm{cmol}_{\mathrm{c}} \cdot \mathrm{kg}^{-1} ; \mathrm{Na}^{+}=0,08 \mathrm{cmol}_{\mathrm{c}} \cdot \mathrm{kg}^{-1} ; \mathrm{P}=11$ mg.kg ${ }^{-3} ; \mathrm{K}=148$ mg.kg ${ }^{-3} ; \mathrm{C}=13$ g.kg-1 $\mathrm{Fe}=162 \mathrm{mg} \cdot \mathrm{dm}^{-3} ; \mathrm{Cu}=0,8 \mathrm{mg} \cdot \mathrm{dm}^{-3}$; $\mathrm{Zn}=66 \mathrm{mg} \cdot \mathrm{dm}^{-3}$ e $\mathrm{Mn}=11,5 \mathrm{mg} \cdot \mathrm{dm}^{-3}$. Foi realizada calagem conforme recomendação do Manual de Adubação do Estado do Rio de Janeiro (De-Polli \& Almeida, 1988), com $700 \mathrm{~kg} \cdot \mathrm{ha}^{-1}$ de calcário com $85 \%$ PRNT e 12,1 \% CaO, aplicando-se metade do corretivo antes da aração e metade antes da gradagem.

As mudas de mandioquinha-salsa (Arracacia xanthorrhiza Bancroft.), cultivar Amarela de Carandaí, que normalmente são cultivadas na região, foram provenientes do próprio local. Os rebentos para a formação das mudas foram selecionados de touceiras de plantas adultas que já haviam terminado os ciclos vegetativos, de onde foram destacados e cortados em bisel, padronizando-se assim o tamanho das mudas. Estas foram plantadas em canteiros de pré- enraizamento, onde permaneceram até os 60 dias, quando foram transplantados no local definitivo. No préenraizamento a umidade do solo foi mantida em aproximadamente $3 / 4$ da capacidade de campo, e não foi aplicado nenhum agrotóxico ou fertilizante neste período.

No transplantio das mudas foram aplicadas no solo as doses de 0,60 e $120 \mathrm{~kg} \cdot \mathrm{ha}^{-1}$ de nitrogênio; 0,150 e 300 kg.ha-1 de $\mathrm{P}_{2} \mathrm{O}_{5}$; e 0 , 80 e $160 \mathrm{~kg} \cdot \mathrm{ha}^{-1}$ de $\mathrm{K}_{2} \mathrm{O}$, usando como fontes uréia, superfosfato simples e cloreto de potássio, respectivamente. Também se aplicou no solo 15 kg.ha-1 de bórax $(10 \%$ de B), 5 kg.ha- ${ }^{-1}$ de sulfato de zinco $(23 \%$ de $\mathrm{Zn}$ ) e 5 t.ha ${ }^{-1}$ de esterco bovino curtido. Os fertilizantes minerais e o orgânico foram distribuídos nas leiras e incorporados manualmente no solo na profundidade de $0-10 \mathrm{~cm}$. A adubação nitrogenada foi parcelada, sendo metade aplicada no transplantio das mudas e outra metade, em cobertura 60 dias após o transplantio. Utilizou-se, durante todo o ciclo, um sistema de irrigação por aspersão, com o objetivo de manter a umidade do solo próxima à capacidade de campo. O delineamento experimental foi de blocos ao acaso com quatro repetições e 27 tratamentos (fatorial 3x3x3) com parcelas de $2,8 \times 4,0$ m e 40 plantas por parcela, com espaçamento de $0,7 \times 0,4 \mathrm{~m}$. Foram descartadas as linhas externas de plantas, permanecendo 18 plantas na área útil de cada parcela. Efetuaram-se quatro capinas manuais e adicionou-se palha de capim como cobertura morta para auxiliar no controle de plantas invasoras e a manutenção da umidade.

Foram realizadas quatro coletas de material vegetal durante o ciclo, aos 90, 150,210 e 300 dias após o transplantio (DAT), sendo coletadas quatro plantas por parcela e separando-se em folhas (limbo + pecíolos), propágulo (coroa e rebentos) e raízes, nas quais foram avaliados os teores de nutrientes na massa seca. O material foi fragmentado e seco em estufa de circulação forçada de ar a 55-65 ${ }^{\circ} \mathrm{C}$ por no mínimo 72 horas. Em seqüência, o material foi finamente moído em moinho de facas com peneira de $2 \mathrm{~mm}$, para posterior análise química. Para o propágulo aos 90 dias após 
o transplante não foi realizada análise dos teores devido à pequena quantidade de material para as determinações. As determinações dos teores de macronutrientes e micronutrientes das plantas foram realizadas conforme a metodologia descrita em Tedesco et al. (1995).

Os dados foram submetidos à análise de variância e, quando se identificou significância pelo teste $\mathrm{F}$, as médias foram comparadas pelo teste Duncan a 5\% de probabilidade, trabalhando-se com o auxílio do programa estatístico Saeg.

\section{RESULTADOS E DISCUSSÃO}

A produtividade média de raízes comerciais foi de aproximadamente 12 t.ha- ${ }^{-1}$, e não se verificou aumento significativo de produção com o aumento das doses de nutrientes. Portz et al. (2003), verificaram que a produção de raízes comerciais aos 300 DAT, não foi influenciada pelas doses crescentes de fósforo e potássio aplicados no plantio e apresentou correlação negativa com as doses de nitrogênio, ou seja, a maior dose promoveu um decréscimo da produção de raízes comerciais. Efeitos negativos da fertilização com nitrogênio na produção de raízes comerciais também foram observadas por Silva et al. (1966). No comportamento do acúmulo de massa seca na planta e suas partes (Figura 1) observa-se a tendência de rápido crescimento em massa seca do propágulo e raízes após os 150 DAT e a tendência quadrática do acúmulo de massa da parte aérea até o final do ciclo, indicando que o início da senescência da planta situa-se em torno dos seis meses de ciclo, fato este também observado por Câmara (1984); Câmara et al. (1985) e Vieira (1995).

Não houveram correlações significativas entre as massas frescas e secas das partes da planta e a produção de raízes comerciais, exceto aos 300 DAT, quando se observaram correlações significativas $(\mathrm{P} \leq 0,01)$ entre a massa seca do propágulo e massa fresca das folhas com a produção de raízes comerciais; 0,46 e 0,45 , respectivamente (Portz, 2001). Bustamante (1988) encontrou correlações positivas entre a produção da parte subterrânea e a parte aérea, indicando que a planta não beneficiou a

Tabela 1. Correlação entre os acúmulos de nutrientes $\left(\mathrm{g}_{\text {planta }}{ }^{-1}\right)$ nas raízes e a produção de raízes comerciais, em kg ha-1 , aos 300 DAT. Seropédica, UFRRJ, 2001.

\begin{tabular}{lc}
\hline Acúmulos de nutrientes nas raízes & Produção de raízes comerciais \\
\hline Nitrogênio & $0,71^{* *}$ \\
Fósforo & $0,68^{* *}$ \\
Potássio & $0,79^{* *}$ \\
Cálcio & $0,44^{* *}$ \\
Magnésio & $0,36^{*}$ \\
Enxofre & $0,61^{* *}$ \\
\hline
\end{tabular}

*Significativo pelo teste $\mathrm{T}$, ao nível de $5 \%$ de probabilidade; **Significativo pelo teste T, ao nível de $1 \%$ de probabilidade.

Tabela 2. Acúmulos médios de macronutrientes (g.planta $\left.{ }^{-1}\right)$ e micronutrientes (mg.planta $\left.{ }^{-1}\right)$ nas folhas, no propágulo e nas raízes, durante o ciclo da mandioquinha-salsa. Seropédica, UFRRJ, 2001.

\begin{tabular}{|c|c|c|c|c|}
\hline & \multicolumn{4}{|c|}{ Acúmulos de nutrientes pelas folhas } \\
\hline & 90 dias & 150 dias & 210 dias & 300 dias \\
\hline $\mathrm{N}$ & $1,28 \mathrm{c}$ & $6,66 b$ & $8,61 a$ & $0,65 d$ \\
\hline $\mathrm{P}$ & $0,16 c$ & $1,75 a$ & $1,64 b$ & $0,20 c$ \\
\hline $\mathrm{K}$ & $2,26 c$ & $20,32 a$ & $12,99 \mathrm{~b}$ & $1,01 d$ \\
\hline $\mathrm{Ca}$ & $0,47 c$ & $3,75 a$ & $3,18 b$ & $0,30 \mathrm{c}$ \\
\hline Mg & $0,20 c$ & $0,89 b$ & $1,20 \mathrm{a}$ & $0,11 d$ \\
\hline S & $0,94 c$ & $7,47 a$ & $4,88 b$ & $0,34 d$ \\
\hline $\mathrm{Mn}$ & $1,25 c$ & $3,66 a$ & $2,76 b$ & $0,16 \mathrm{~d}$ \\
\hline $\mathrm{Fe}$ & $6,19 c$ & $22,78 b$ & $49,96 a$ & $12,27 \mathrm{c}$ \\
\hline $\mathrm{Zn}$ & $0,39 c$ & $1,27 a$ & $1,06 \mathrm{~b}$ & $0,08 d$ \\
\hline \multirow[t]{3}{*}{$\mathrm{Cu}$} & $0,08 b$ & $0,40 a$ & $0,42 a$ & $0,02 c$ \\
\hline & \multicolumn{4}{|c|}{ Acúmulos de nutrientes pelos propágulos } \\
\hline & 90 dias & 150 dias & 210 dias & 300 dias \\
\hline $\mathrm{N}$ & -- & $1,60 \mathrm{c}$ & $3,83 b$ & $5,19 a$ \\
\hline $\mathrm{P}$ & -- & $0,57 c$ & $1,36 a$ & $1,11 \mathrm{~b}$ \\
\hline $\mathrm{K}$ & -- & $5,50 \mathrm{c}$ & $8,99 a$ & $8,06 b$ \\
\hline $\mathrm{Ca}$ & - & $0,70 b$ & $1,55 \mathrm{a}$ & $1,70 \mathrm{a}$ \\
\hline $\mathrm{Mg}$ & -- & $0,39 c$ & $1,31 \mathrm{a}$ & $1,06 b$ \\
\hline S & -- & $2,70 \mathrm{c}$ & $6,10 a$ & $4,19 b$ \\
\hline $\mathrm{Mn}$ & -- & $1,07 \mathrm{c}$ & $1,63 a$ & $1,29 b$ \\
\hline $\mathrm{Fe}$ & -- & $19,02 c$ & $70,70 a$ & $49,80 b$ \\
\hline $\mathrm{Zn}$ & -- & $0,80 \mathrm{c}$ & $2,00 a$ & $1,55 b$ \\
\hline \multirow[t]{3}{*}{$\mathrm{Cu}$} & - & $0,18 \mathrm{c}$ & $0,41 \mathrm{a}$ & $0,25 b$ \\
\hline & \multicolumn{4}{|c|}{ Acúmulos de nutrientes pelas raízes } \\
\hline & 90 dias & 150 dias & 210 dias & 300 dias \\
\hline $\mathrm{N}$ & $0,04 d$ & $0,53 c$ & $2,44 b$ & $3,77 a$ \\
\hline$P$ & $0,01 \mathrm{c}$ & $0,21 b$ & $1,20 a$ & $1,25 a$ \\
\hline $\mathrm{K}$ & $0,06 \mathrm{~d}$ & $2,08 \mathrm{c}$ & $8,67 b$ & $10,94 a$ \\
\hline $\mathrm{Ca}$ & $0,003 b$ & $0,16 b$ & $1,02 a$ & $0,89 a$ \\
\hline $\mathrm{Mg}$ & $0,01 \mathrm{c}$ & $0,11 b$ & $0,52 a$ & $0,52 a$ \\
\hline S & $0,02 d$ & $0,89 c$ & $4,10 \mathrm{~b}$ & $6,16 a$ \\
\hline $\mathrm{Mn}$ & $0,07 d$ & $0,26 b$ & $0,61 \mathrm{a}$ & $0,15 c$ \\
\hline $\mathrm{Fe}$ & $0,61 \mathrm{c}$ & $2,12 \mathrm{c}$ & $14,53 a$ & $6,63 b$ \\
\hline $\mathrm{Zn}$ & $0,01 d$ & $0,12 \mathrm{c}$ & $0,63 a$ & $0,44 b$ \\
\hline $\mathrm{Cu}$ & $0,004 d$ & $0,07 c$ & $0,23 a$ & $0,16 b$ \\
\hline
\end{tabular}

*Médias seguidas de mesma letra, nas linhas, não diferem significativamente pelo teste Duncan a $5 \%$.

porção foliar em detrimento da produção de raízes. Câmara et al. (1985) e
Vieira (1995) observaram correlação positiva entre a produção da parte sub- 
Tabela 3. Teores médios de macronutrientes $\left(\mathrm{g} .100 \mathrm{~g}^{-1}\right)$ e micronutrientes $\left(\mathrm{mg} \cdot 100 \mathrm{~g}^{-1}\right)$ nas folhas, no propágulo e nas raízes, durante o ciclo da mandioquinha-salsa. Seropédica, UFRRJ, 2001.

\begin{tabular}{|c|c|c|c|c|}
\hline & \multicolumn{4}{|c|}{ Teores de nutrientes pelas folhas } \\
\hline & 90 dias & 150 dias & 210 dias & 300 dias \\
\hline $\bar{N}$ & $3,43 a$ & $2,16 \mathrm{~d}$ & $2,84 b$ & $2,32 \mathrm{c}$ \\
\hline$P$ & $0,42 c$ & $0,57 a$ & $0,54 b$ & $0,37 d$ \\
\hline K & $5,98 b$ & $6,65 a$ & $4,33 c$ & $3,71 d$ \\
\hline $\mathrm{Ca}$ & $12,13 a$ & $11,81 a$ & $10,46 b$ & $9,96 b$ \\
\hline $\mathrm{Mg}$ & $5,21 a$ & $2,89 d$ & $3,96 b$ & $3,61 \mathrm{c}$ \\
\hline$S$ & $24,89 a$ & $24,36 a$ & $16,20 \mathrm{~b}$ & $11,29 c$ \\
\hline $\mathrm{Mn}$ & $32,55 a$ & $11,64 b$ & $9,13 c$ & $4,32 d$ \\
\hline $\mathrm{Fe}$ & $174,01 b$ & $72,25 c$ & $153,36 b$ & $294,58 a$ \\
\hline $\mathrm{Zn}$ & $10,29 a$ & $4,09 b$ & $3,51 \mathrm{c}$ & $2,41 \mathrm{~d}$ \\
\hline \multirow[t]{3}{*}{$\mathrm{Cu}$} & $2,05 a$ & $1,26 b$ & $1,40 \mathrm{~b}$ & $0,74 \mathrm{c}$ \\
\hline & \multicolumn{4}{|c|}{ Teores de nutrientes pelos propágulos } \\
\hline & 90 dias & 150 dias & 210 dias & 300 dias \\
\hline $\mathrm{N}$ & - & $1,54 a$ & $1,44 b$ & $1,51 a$ \\
\hline$P$ & - & $0,54 a$ & $0,52 b$ & $0,32 \mathrm{c}$ \\
\hline K & -- & $5,27 a$ & $3,45 b$ & $2,35 \mathrm{c}$ \\
\hline $\mathrm{Ca}$ & -- & $6,37 a$ & $5,83 a b$ & $5,06 b$ \\
\hline $\mathrm{Mg}$ & -- & $3,76 b$ & $4,99 a$ & $3,05 c$ \\
\hline$S$ & -- & $25,58 a$ & $23,07 \mathrm{~b}$ & $12,53 \mathrm{c}$ \\
\hline $\mathrm{Mn}$ & -- & $10,18 a$ & $6,24 b$ & $3,73 c$ \\
\hline $\mathrm{Fe}$ & -- & $170,68 b$ & $279,19 a$ & $142,60 \mathrm{c}$ \\
\hline $\mathrm{Zn}$ & - & $7,47 a$ & $7,49 a$ & $4,54 b$ \\
\hline \multirow[t]{3}{*}{$\mathrm{Cu}$} & -- & $1,66 a$ & $1,49 a$ & $0,71 \mathrm{c}$ \\
\hline & \multicolumn{4}{|c|}{ Teores de nutrientes pelas raízes } \\
\hline & 90 dias & 150 dias & 210 dias & 300 dias \\
\hline $\mathrm{N}$ & $1,90 a$ & $0,54 \mathrm{c}$ & $0,52 c$ & $0,62 b$ \\
\hline$P$ & $0,30 a$ & $0,21 \mathrm{c}$ & $0,26 b$ & $0,20 c$ \\
\hline K & $3,10 a$ & $1,99 b$ & $1,87 \mathrm{c}$ & $1,80 c$ \\
\hline $\mathrm{Ca}$ & 2,91-- & $1,68--$ & $2,28--$ & $1,52--$ \\
\hline $\mathrm{Mg}$ & $5,57 a$ & $1,14 b$ & $1,11 \mathrm{~b}$ & $0,85 b$ \\
\hline S & $10,19 a$ & $7,71 b$ & $8,34 b$ & $10,15 a$ \\
\hline $\mathrm{Mn}$ & $37,25 a$ & $2,65 b$ & $1,30 \mathrm{bc}$ & $0,25 c$ \\
\hline $\mathrm{Fe}$ & $351,79 a$ & $20,10 b$ & $31,11 b$ & $11,32 b$ \\
\hline $\mathrm{Zn}$ & $6,10 a$ & $1,19 b$ & $1,35 b$ & $0,71 \mathrm{c}$ \\
\hline $\mathrm{Cu}$ & $2,31 a$ & $0,74 b$ & $0,50 b c$ & $0,25 c$ \\
\hline
\end{tabular}

*Médias seguidas de mesma letra, nas linhas, não diferem significativamente pelo teste Duncan a $5 \%$.

terrânea e da parte aérea, ou seja, as plantas de mandioquinha-salsa mais altas, que foram as mais exuberantes e possuíam a maior área foliar, produziram maior quantidade de raízes comerciais.

Não foram encontradas correlações significativas entre os teores de nutrientes (N, P, K, Ca, Mg, S, Fe, Zn, Cu e $\mathrm{Mn}$ ) das partes da planta, nas épocas de amostragens, com a produção de raízes comerciais aos 300 DAT. No entanto, observaram-se correlações significativas entre o acúmulo de $\mathrm{N}, \mathrm{P}, \mathrm{K}, \mathrm{Ca}, \mathrm{Mg}$ e $S$ nas raízes e a produção de raízes comerciais nos 300 DAT (Tabela 1).

Os acúmulos médios de macro e micronutrientes nas folhas, propágulos e raízes, durante o ciclo das plantas, são apresentados na tabela 2 .

Verificou-se, aos 300 DAT, que as raízes, propágulos e folhas tiveram os maiores acúmulos de nutrientes, respectivamente nesta ordem. Mesquita Filho e Souza (1996) verificaram, aos 11 meses, maiores acúmulos de $\mathrm{P}, \mathrm{K}, \mathrm{Cu}$ e $\mathrm{Zn}$ no propágulo, apesar dos teores se apresentarem maiores nas folhas, propágulo e raízes, respectivamente.
Como a raiz é o órgão preferencial de reserva na planta e, portanto, o de maior massa seca aos 300 DAT, observaram-se maiores acúmulos de nutrientes neste órgão, quando comparado às outras épocas. Segundo Câmara (1984), a planta é estruturada de modo a ter suas reservas na parte subterrânea menos sujeitas a variações climáticas e ao ataque de insetos, garantindo energia para sua fase reprodutiva, considerando o longo período desde sua instalação até a produção de sementes, que é de 24 meses.

Observou-se que no acúmulo de nutrientes nas folhas (Tabela 2) as maiores médias estão entre os 150 e 210 DAT, período de maior exuberância da parte aérea da planta, e que depois decaem drasticamente pela senescência e perda das folhas no final do ciclo vegetativo. Nos propágulos verificaram-se os maiores acúmulos aos 210 DAT, exceto para o nitrogênio que foi aos 300 DAT (Tabela 2) o que pode estar relacionado de alguma forma com o mecanismo de translocação de reservas para as raízes no final do ciclo da planta. Nas raízes observaram-se maiores acúmulos de nutrientes aos 210 e 300 DAT (Tabela 2), indicando nitidamente ser um órgão preferencial de reserva da planta, quando esta se aproxima do final do ciclo vegetativo. Lima et al. (1985), verificaram que as maiores quantidades acumuladas de N, P, K e Zn estavam presentes nos propágulos; enquanto $\mathrm{Ca}, \mathrm{Mg}, \mathrm{Cu}$ e $\mathrm{Mn}$, nas raízes e Fe nas folhas. No entanto, os acúmulos podem ser influenciados pelos tratamentos aplicados ao solo no plantio, a época de coleta, a cultivar cultivada e condições de manejo da cultura.

Os teores de nutrientes na planta geralmente estão relacionados com os acúmulos, apesar de neste trabalho não terem se correlacionado com a produção de raízes comerciais. Houve uma tendência de queda dos teores nas folhas ao longo do tempo (Tabela 3), exceto para o teor de ferro que apresentou um crescimento. A grande queda de massa das folhas no final do ciclo e a translocação de assimilados para os drenos da planta (propágulos e raízes) possivelmente influenciaram na queda dos teores de nutrientes neste órgão. Também se observa queda dos teores no 
propágulo com o tempo (Tabela 3), mas neste caso, pode estar relacionada ao efeito de diluição devido ao grande acúmulo de massa neste órgão no final do ciclo. $\mathrm{O}$ mesmo pode ser verificado para os teores nas raízes (Tabela 3). Câmara (1984), avaliando níveis de cálcio, magnésio potássio e fósforo, dos quatro aos 11 meses de ciclo da planta, observou que não houve grande variação dos teores de nutrientes no tempo.

A mandioquinha-salsa, considerada rústica por não responder a adubação e ser resistente, possui um mecanismo fisiológico complexo e ainda pouco estudado. Responde de forma diferenciada as condições de manejo, podendo acumular quantidades consideráveis de nutrientes (Ortiz \& Acin, 1997; Ortiz et al., 1998) quando disponibilizados no solo, apesar do acúmulo nem sempre refletir em maiores produtividades. Deve-se considerar ainda a adaptabilidade dos clones cultivados nos locais de cultivo e os tratos culturais aplicados na planta ao longo do ciclo.

Apesar dos resultados não mostrarem respostas conclusivas, pode-se dizer que a planta possui uma grande demanda de nutrientes a partir dos 150 DAT, período de maior desenvolvimento das folhas e início de grande acúmulo no propágulo e raízes, o que pode influenciar a produção de raízes comerciais no final do ciclo da planta.

\section{AGRADECIMENTOS}

Agradecemos aos colegas de graduação do curso de Agronomia pela ajuda nos trabalhos de campo e no laborató- rio, ao Departamento de Solos da UFRRJ pelas dependências e equipamentos utilizados e a FAPERJ pelo apoio financeiro e bolsa de estudos.

\section{REFERÊNCIAS}

BUSTAMANTE PG. 1988. Melhoramento de batata-baroa (Arracacia xanthorrhiza Bancroft). I. Biologia floral: obtenção e caracterização de novos clones, correlações genéticas. Viçosa: UFV. 94p. (Tese mestrado).

CÂMARA FLA. 1984. Estudo de tecnologias objetivando precocidade de produção de batata-baroa (Arracacia xanthorrhiza Bancroft). Viçosa: UFV. 50p. (Tese mestrado).

CÂMARA FLA. 1990. Sintomatologia de carências de macronutrientes e boro em mandioquinha-salsa (Arracacia xanthorrhiza Bancroft). Piracicaba: USP-ESALQ. 66p. (Tese doutorado).

CÂMARA FLA; MAFFIA LM; CASALI VWD. 1985. Curva de crescimento e utilização da mandioquinha-salsa na alimentação. Horticultura Brasileira 3: 29-33.

DEL VALLE JUNIOR R, ORTIZ CE, SANTIAGO-CORDOVA MA. 1995. Fertilization of arracacha in na ultissol. Journal of Agriculture of the University of Puerto Rico 79: 3-4.

DE-POLLI H; ALMEIDA DL. 1988. Manual de adubação para o Estado do Rio de Janeiro. Rio de Janeiro: UFRRJ. 179p.

EMBRAPA. Centro Nacional de Pesquisa de Solos. Sistema Brasileiro de Classificação de Solos. Brasília : Embrapa Produção de informação; Rio de Janeiro : Embrapa Solos, 1999. 412 p.

LIMA LA, ZANIN ACW, MISCHAN MM. 1985. Acúmulo de matéria seca e de nutrientes por plantas de mandioquinha-salsa colhida dos nove aos doze meses de plantio. Horticultura Brasileira 3: 76.

MESQUITA FILHO MV, SOUZA AF. 2000 Acúmulo de matéria seca e de nutrientes na cultura de mandioquinha-salsa em solo do cerrado. IN: REUNIÃO BRASILEIRA DE FERTILIDADE DO SOLO E NUTRIÇÃO DE PLANTAS, 22. Manaus. Embrapa/CNPH-hortaliças.
MESQUITA FILHO MV, SOUZAAF, SILVAHR, SANTOS FF, OLIVEIRA AS. 1996. Adubação nitrogenada e fosfatada para a produção comercializável de mandioquinha-salsa em Latossolo Vermelho-Amarelo. Horticultura Brasileira 14: 2.

ORTIZ CE; ACIN NM. 1997. Estimate of macronutrients uptake by arracacha at harvest. San Juan: University of Puerto Rico. $7 \mathrm{p}$.

ORTIZ CE; ORENGO-SANTIAGO E; ACIN NM. 1998. Dry weight accumulation and nutrient uptake by arracacha grown under controlled conditions. San Juan: University of Puerto Rico. 9p.

PEREIRA AS. 1995. O valor nutritivo da mandioquinha-salsa. In: ENCONTRO NACIONAL DE MANDIOQUINHA-SALSA, 5. Palestras e trabalhos técnicos... Venda Nova do Imigrante: SOB. p.14-16.

PORTZ A. 2001. Determinação de parâmetros nutricionais e produtivos da cultura de mandioquinha-salsa em Nova Friburgo-RJ. Rio de Janeiro: UFRRJ. 99p. (Tese mestrado).

PORTZA; MARTINS CAC; LIMA E. 2003. Crescimento e produção de raízes comercializáveis de mandioquinha-salsa em resposta à aplicação de nutrientes. Horticultura Brasileira 21: 485-488.

SILVA JR, GARCIA BH, NORMANHA ES, FREIRE ES. 1966. Efeito de doses crescentes de nitrogênio, fósforo e potássio sobre a produção de raízes de mandioquinha-salsa. Bragantia 25: 365-370.

TEDESCO MJ; GIANELLO C; BISSANI CA, BOHNEN H; VOLKWEISS SJ. 1995. Análise de solo, plantas e outros materiais. 2 ed., Porto Alegre: UFRGS. 174p.

VIEIRA MC. 1995. Avaliação do crescimento e da produção de clones e efeito de resíduo orgânico e de fósforo em mandioquinha-salsa no Estado do Mato Grosso do Sul. Viçosa. Viçosa: UFV. 146p. (Tese doutorado).

ZÁRATE NAH, VIEIRA MC, ARAÚJO C. 2001. Perdas de peso no armazenamento de raízes de mandioquinha-salsa 'Amarela de Carandaí': Efeitos de tratamentos no cultivo a após a colheita. Ciências Agrotécnicas 25: 1337-1342. 